\title{
The Right to Privacy of Personal Information and Private Life (Privacy): The Issues Nowadays
}

\author{
Irisi Topalli \\ Professor at the Faculty of Law, University of North "Argenti", \\ Shkoder, Albania, E-mail: iris_topalli@hotmail.com
}

\section{Doi:10.5901/mjss.2013.v4n10p648}

\section{Abstract}

\begin{abstract}
The right to privacy of personal information and private life, otherwise known as privacy, is an important right of ours, which despite dating years ago, in the European Convention on Human Rights in 1950, is today faced with difficulties and problems in various legal and social plan. So, first of all, today there are still countries that do not have this right expressly provided in their constitution. Also, despite different national and international acts (especially comunitare acts), are still not very clear instruments for the protection of this right. This has made the role of jurisprudence to be fundamental in this regard, based on discretionary and subjective criterion of judicial authority. Also today, in the era of internet and technology, more and more users want to know which of their data are collected and where they go. Hence the need for new legislation aimed to rescue the users' confidence in online services. Another problem is the one that has to do with simplification of norms in the area of privacy and standardization of procedures among different states, thus eliminating the differences between national legislations, which are the first cause of uncertainty. The Problematic is even safeguard this right, when dealing with other constitutional values. Delicate in this regard, is its relationship with the right of defense, the publication of the decision, and the right to information. All these will be subject to the treatment of this topic, given the importance and the problems that they present today.
\end{abstract}

\section{Premessa}

Si discute molto della privacy ai giorni d’oggi. Ma che cos'è la privacy? Quella che con un termine ormai entrato nell'uso comune viene indicata come privacy è il diritto alla riservatezza delle informazioni personali e della propria vita private. Le problematiche della privacy sono tante e sicuramente non e possibile di studiarle tutte in questo studio, pero ci espanderemo notevolmente in alcuni elementi importanti come la legislazione in materia di privacy, quando è iniziato come un concetto giuridico, quali risorse legali e quale ruolo è stato giocato dalla giurisprudenza come fonte, approfondendo di più nella legislazione italiana; la privacy e internet; il diritto della privacy e gli altri diritti costituzionali nella legislazione italiana.

\section{La legislazione sulla privacy}

Nella società di oggi, sta diventando sempre più possibile la violazione della privacy, a causa dello sviluppo di tecnologie e strumenti che non permettono all'uomo di sviluppare pienamente la sua sfera di vita privata ${ }^{1}$.Quella che con un termine ormai entrato nell'uso comune viene indicata come privacy è il diritto alla riservatezza delle informazioni personali e della propria vita privata, cioè uno strumento posto a salvaguardia e a tutela della sfera privata del singolo individuo, da intendere come la facoltà di impedire che le informazioni riguardanti tale sfera personale siano divulgate in assenza dell'autorizzazione dell'interessato, od anche il diritto alla non intromissione nella sfera privata da parte di terzi. Tale diritto assicura all'individuo il controllo su tutte le informazioni e i dati riguardanti la sua vita privata, fornendogli nel contempo gli strumenti per la tutela di queste informazioni².

L'istituto nasce come diritto a the right to be let alone ("il diritto di essere lasciati in pace"), secondo la formulazione del giurista statunitense Louis Brandeis che fu probabilmente il primo al mondo a formulare una legge sulla riservatezza, insieme a Samuel Warren (The Right to Privacy, "Harvard Law Review",1890)3. A ben vedere l'opera dei

\footnotetext{
1 Omari, L. \& Anastasi, A., (2010), E Dejta Kushtetuese, ABC, 147-148

2 http://brunosaetta.it/privacy/privacy-o-diritto-alla-riservatezza-e-protezione-dei-dati-personali.html

3 https://it.wikipedia.org/wiki/Privacy\#Fonti_comunitarie
} 
due giuristi bostoniani non rappresenta tutta via una creazione ex novo, ma semmai una rifondazione di un diritto già noto e tutelato, benché secondo logiche differentit.

Sempre più compresso nella società della comunicazione elettronica, nel tempo si è evoluto ed oggi si parla di privacy non più, e non solo, nel senso di protezione dei dati personali (quindi come diritto negativo volto a impedire la rilevazione di informazioni sul nostro conto), ma in una accezione più ampia anche quale diritto ad esprimere liberamente le proprie aspirazioni più profonde e realizzarle attingendo liberamente alla proprie potenzialità.

I primi riferimenti alla privacy si possono far risalire alla Convenzione Europea dei Diritti dell'Uomo dell 1950, che già stabiliva come non può esservi ingerenza di una autorità pubblica nell'esercizio del diritto alla propria libertà individuale, a meno che tale ingerenza sia prevista dalla legge in quanto misura necessaria per la sicurezza nazionale, per la pubblica sicurezza, per il benessere economico del paese, per la difesa dell'ordine e per la prevenzione dei reati, per la protezione della salute 0 della morale, 0 per la protezione dei diritti e delle libertà altrui ${ }^{5}$. Questo fondamentale concetto è stato poi riportato ed espanso in vari accordi internazionali, come ad esempio quello di Schengen, ed anche nella Carta dei Diritti Fondamentali dell'Unione Europea che all'art. 8 così recita: "1. Ogni individuo ha diritto alla protezione dei dati di carattere personale che lo riguardano. 2. Tali dati devono essere trattati secondo il principio di lealtà, per finalità determinate e in base al consenso della persona interessata 0 a un altro fondamento legittimo previsto dalla legge. Ogni individuo ha il diritto di accedere ai dati raccolti che lo riguardano e di ottenerne la rettifica. 3. Il rispetto di tali regole è soggetto al controllo di un'autorità indipendente"6.

Infatti, sull'esistenza di un diritto alla privacy garantito dalla costituzione, ci sono state opinioni diverse nella dottrina giuridica. Questo ha fatto che il diritto di privacy venisse esplicitamente sanzionato un po 'più tardi nei primi costituzioni, e formulato nelle nuove costituzioni. In alcuni dei primi costituzioni (per esempio nella Costituzione italiana), non c'è un articolo diretto a garantire tale diritto, tuttavia, anche in quelle, tale conclusione si raggiungie da alcune altre formulazioni. Per esempio, fino al 1997 non esistevano nel ordinamento italiano norme specifiche in materia. A questo riguardo possiamo menzionare anche la Costituzione albanese, la quale non sanciona esplicitamente tale diritto, ma fa riferimento alla privacy tramite altri diritti fondamentali dell uomo ${ }^{7}$. Colsi l'articolo 35 della Costituzione albanese prevede: "nessuno non si puo obligare, ecetto quando la legge lo chiede, di fare publiche i suoi dati personali"8.

I sistemi di Common Law hanno individuato agevolmente l'esistenza e poi la disciplina del Right to Privacy, data la peculiare funzione esercitata dalla giurisprudenza in tali sistemi.

Per contro, la vicenda del diritto alla riservatezza nell'esperienza italiana costituisce un esempio di "atrofia" dell'apparato legislativo in senso proprio: infatti il Legislatore si è dimenticato di disciplinare la materia, favorendo così il permanere di una situazione di incertezza. A tale anomalia ha dovuto supplire, come e' ormai consuetudine, la giurisprudenza, la quale, almeno apparentemente, si è costituita fonte di diritto ${ }^{9}$.

Per concretizzare ciò che è stato scritto sopra, questo studio si espanderà un po 'di più nella legislazione italiana, portando due principali difensori del diritto della privacy.

II primo è il Codice in Materia di Protezione dei Dati Personali (Codice della privacy). Inizialmente, la riservatezza era più che altro un diritto delle persone famose, infatti I'Italia arrivò come penultima in Europa ad approvare una legge di tutela della privacy di applicazione generale, trasfusa prima nella legge 675 del 1996 e poi nel Codice in Materia di Protezione dei Dati Personali (Codice della privacy) cioè il Decreto legislativo 30 giugno 2003, n. 196, dal quale si evince chiaramente che la privacy non è solo il diritto a non vedere trattati i propri dati senza consenso, ma anche l'adozione di cautele tecniche ed organizzative che tutti, compreso le persone giuridiche, devono rispettare per procedere in maniera corretta al trattamento dei dati altrui. Detta normativa, considerata la più completa a livello europeo, dedica la prima parte ai principi generali, dettando le definizioni essenziali per la comprensione della normativa, tra le quali quelle di dato personale e di trattamento.

Mentre l'organo preposto al controllo relativo alla corretta applicazione della normativa in materia di privacy, è il Garante per la Protezione dei Dati Personali, autorità amministrativa collegiale ed indipendente, i cui membri sono nominati dal Parlamento e che opera un controllo preventivo e successivo sulle attività di trattamento di dati personali svolte in Italia. II Garante, che opera autonomamente dal Governo, ha poteri istruttori, consultivi e sanzionatori, e

\footnotetext{
${ }^{4}$ Mantelero, A., (2008), Contratto e impresa, Dialoghi con la giurisprudenza civile e commercial, CEDAM, 758- 759

$5 \mathrm{http}$ ://brunosaetta.it/privacy/privacy-o-diritto-alla-riservatezza-e-protezione-dei-dati-personali.html

${ }^{6}$ Carta dei Diritti Fondamentali dell'Unione Europea

7 Omari, L. \& Anastasi, A., (2010), E Dejta Kushtetuese, ABC, 147-148

\&La Costituzione Albanese, 1998

${ }^{9}$ Cardaci, F. \& Olivetti, S., (1997), Diritto Civile, II diritto alla riservatezza in Italia
} 
costituisce il primo grado per il ricorso amministrativo contro eventuali violazioni della normativa. Eventuali decisioni del Garante, assunte in contraddittorio con le parti in causa, sono impugnabili dinanzi alla magistratura ${ }^{10}$.

\section{Privacy e Internet}

E parlando di questo, il problema di garantire la privacy si pone soprattutto in maniera pressante in internet, dove la diffusione dei dati è facile e veloce. Inoltre, tale problema è strettamente legato al tema della sicurezza informatica, visto che spesso si verificano furti di dati attraverso la rete.

Dal punto di vista giuridico si è, infatti, sentita l'esigenza di ampliare il vecchio ordinamento giuridico e, di conseguenza, anche la normativa relativa al concetto di privacy che, fino a non molti anni fa, si occupava esclusivamente della tradizionale corrispondenza e della comunicazione telegrafica e telefonica ${ }^{11}$.

Nell Febbraio 2012, il commissario per la Giustizia alla Commissione Europea, Viviane Reding, ha presentato la riforma della normativa europea sulla privacy. Già nel novembre del 2010 l'Europa si era posta il problema della protezione dei dati personali, e in una comunicazione definiva la strategia e gli obiettivi di una riforma radicale della privacy. La direttiva in materia di privacy del 1995, infatti, risale ad un periodo nel quale l'uso della rete non era così diffuso e pervasivo, da cui l'esigenza di affrontare e risolvere le problematiche sorte a seguito della proliferazione di social network e servizi transnazionali che macinano quotidianamente quantità enormi di dati personali, con le correlate difficoltà per gli utenti di comprendere esattamente quali diritti hanno e a quali legislazioni fare riferimento.

L'intenzione è quella di realizzare una normativa che sappia contemperare equamente le esigenze delle parti in causa, quindi da una parte dovrà essere meno dispendiosa per i governi e le imprese, dall'altra dovrà essere in grado di garantire maggiore protezione per gli utenti.

Gli obiettivi che si intendono raggiungere sono vari. Innanzitutto la Commissione europea vuole rafforzare i diritti delle persone, in modo che la raccolta e l'uso dei dati personali sia limitato al minimo necessario. Per raggiungere questo risultato si intende promuovere la trasparenza, cioè gli utenti devono essere correttamente e compiutamente informati sui dati raccolti, sul loro uso e sui tempi di utilizzo, in modo che il consenso sia davvero informato. La proposta di riforma prevede, infatti, la necessità di un consenso esplicito da parte del cittadino, prima che le aziende trattino i loro dati, e in linea di massima i dati dovrebbero essere privati per impostazione predefinita. La riforma, inoltre, si ispira ad un principio di correttezza, nel senso che i cittadini non devono essere costretti a condividere i loro dati, ed infine si intende aumentare il controllo dell'utente sui dati, in modo che egli possa decidere in maniera semplice ed efficace cosa condividere.

L'intenzione è quindi di limitare i modelli di business invadenti, così garantendo un livello di protezione maggiore in modo da consentire a tutti i cittadini europei di cogliere le opportunità offerte dalle nuove tecnologie, spazzando i timori di rischi per la propria privacy.

In tal senso verrebbe regolamentato il diritto all'oblio, inteso come il diritto che ogni utente ha alla cancellazione dei propri dati nel momento in cui non sussistano più ragioni legittime per mantenerli e l'utente ne richieda l'eliminazione.

La riforma ovviamente dovrà essere improntata alla chiarezza, alla semplificazione e alla flessibilità. L'intento è di realizzare norme chiare e semplici, facili da comprendere ed attuare, che non diano adito a differenti interpretazioni, in modo che gli operatori del settore non dovranno realizzare procedure alternative per evitare "minacce" di denunce, ma potranno servirsi delle procedure standard. Ovviamente si rende necessario anche ridurre gli oneri amministrativi e burocratici, eliminando quelli inutili per le imprese, tenendo conto della praticabilità delle soluzioni da implementare, con procedure e sanzioni proporzionate alle circostanze evitando effetti intimidatori.

La standardizzazione delle procedure, requisito essenziale della riforma, eliminando le differenze tra le varie legislazioni potrà finalmente portare ad una armonizzazione delle norme e quindi alla realizzazione del mercato unico digitale che sarà di beneficio per tutti. L'intento è di realizzare norme uguali per tutti gli Stati in modo da eliminare le incertezza applicative da parte delle imprese e i dubbi degli utenti sui loro diritti.

In tale prospettiva si prevede un rafforzamento dei poteri di controllo della autorità deputate alla protezione dei dati, migliorandone la cooperazione e il coordinamento. Con la semplificazione le imprese avranno a che fare solo con le autorità di protezione del loro paese di origine, piuttosto che con le autorità di ogni Stato dove operano.

Le norme dovranno necessariamente essere flessibili, cioè in grado di adattarsi a tutti gli Stati e tutte le legislazioni, e non solo UE.

${ }^{10} \mathrm{http}: / /$ brunosaetta.it/privacy/privacy-o-diritto-alla-riservatezza-e-protezione-dei-dati-personali.html

$11 \mathrm{http} / / /$ brunosaetta.it/privacy/privacy-o-diritto-alla-riservatezza-e-protezione-dei-dati-personali.html 
Ovviamente la riforma considera la necessità di conciliare la protezione della privacy con la libertà di espressione nel web, laddove spetterà ai singoli paesi definire le esenzioni alle regole sulla privacy, tramite regole che si applicheranno alla stampa e ai media ${ }^{12}$.

\section{II diritto della privacy e gli altri diritti costituzionali nella legislazione italiana}

I diritti costituzionali, associati di più con il diritto della privacy sono: il diritto all'informazione, il diritto di difesa e il diritto della pubblicazione delle sentenze. Di conseguenza, si vorra solo studiare l'interconnessione tra di loro.

La privacy, intesa nel modo sopra descritto, trova una sua limitazione nel rapporto col diritto di cronaca e il diritto all'informazione costituzionalmente garantito, e si è posto il problema di stabilire il corretto compromesso tra i due interessi. II diritto all'informazione non deve essere inteso soltanto come diritto ad informare, quindi come diritto di cronaca, ma anche come diritto alla manifestazione del pensiero, tutelato dall'articolo 21 della Costituzione, che riguarda non solo i giornalisti ma anche e soprattutto tutti i cittadini.

II bilanciamento tra i due interessi, come sancisce il Codice della privacy in materia di protezione dei dati personali, si realizza attraverso la previsione che il soggetto interessato presti il consenso esplicito al trattamento dei dati che lo riguardano, mantenendo così il controllo su tali informazioni. Tale consenso, in particolare, è anche revocabile.

A chi esercita attività giornalistica è, però, permesso il trattamento dei dati personali, la comunicazione e la diffusione anche senza il consenso dell'interessato e, con riferimento ai dati sensibili e giudiziari, senza la preventiva autorizzazione del Garante per la Protezione dei Dati Personali, purché ci si attenga ai limiti dettati dal diritto di cronaca, individuati nell'essenzialità della notizia e nell'interesse pubblico, con l'unica eccezione dei dati relativi a circostanze 0 fatti resi noti direttamente dall'interessato 0 attraverso i suoi comportamenti pubblici.

Il diritto di difesa, essendo un diritto di rango costituzionale (art. 24 della Costituzione), prevale sul diritto alla privacy e non può essere soggetto a limitazioni essendo posto nell'interesse pubblico.

L'utilizzo di dati personali è, quindi, sempre ammesso in sede giudiziaria, ovviamente purché il diritto che si intende tutelare sia di rango pari a quello dell'interessato. In tale ottica, il diritto alla privacy risulta sovraordinato al diritto d'autore, essendo quest'ultimo rivolto a tutelare meri interessi economici e non diritti e libertà del'individuo, per cui una azienda non è mai autorizzata a tracciare dati dei cittadini al fine di verificare una eventuale violazione dalla normativa sul diritto d'autore, tale attività è infatti demandata in via esclusiva allo Stato e in particolare all'autorità giudiziaria ${ }^{13}$.

Con un provvedimento pubblicato il 4 gennaio nella Gazzetta Ufficiale n. 2 del 2011, il Garante per la Protezione dei Dati Personali ha finalmente riscritto le regole sull'informazione giuridica. In particolare si tratta delle linee guida che regolano la pubblicazione di sentenze e provvedimenti giurisdizionali su riviste giuridiche, $\mathrm{cd}$ e dvd, nonché siti istituzionali, con una particolare attenzione ai minori coinvolti in vicende processuali.

In sintesi, la magistratura, Cassazione, tribunali e corti d'appello, può rilasciare copie integrali delle sentenze ai giornalisti senza oscurare i nomi degli imputati, in quanto le sentenze sono atti pubblici pronunciati "in nome del Popolo Italiano", come ha ricordato la Suprema Corte. Ma volendo pubblicare una sentenza su una rivista di informazione giuridica è necessario oscurare i dati personali. Tale obbligo, però, non vale per la cronaca giudiziaria, poiché quest'ultima deve assicurare il diritto d'informazione dei cittadini, essenziale per il concreto esercizio della sovranità popolare ${ }^{14}$.

\section{Conclusioni e Raccomandazioni}

Nonostante i diversi atti nazionali ed internazionali (in particolare atti comunitare), non sono ancora molto chiari gli strumenti per la tutela di questo diritto.

Il ruolo della giurisprudenza è stata fondamentale perché, in assenza di norme giuridiche esplicite, si è costituita fonte di diritto della privacy.

Anche se il diritto alla privacy non è sancito in modo diretto dalla Costituzione italiana, vi è un codice speciale, il Codice della privacy, che protegge in modo completo tale diritto, e che, a nostro parere, fa che questo diritto sia molto più sancito e protetto che da un unico articolo.

Nonostante gli sforzi dell UE avevano cominciato prima, nel 2012 è stato raggiunto, attraverso la riforma della normativa europea sulla privacy, la semplificazione delle norme in materia di privacy e la standardizzazione delle

\footnotetext{
${ }^{12 h t t p: / / b r u n o s a e t t a . i t / p r i v a c y / p r i v a c y-l a-r i f o r m a-d e l l a-u e-e-i l-d o-n o t-t r a c k-s t a n d a r d . h t m l ~}$

${ }^{13} \mathrm{http}$ ://brunosaetta.it/privacy/privacy-o-diritto-alla-riservatezza-e-protezione-dei-dati-personali.html

${ }^{14} \mathrm{http}: / /$ brunosaetta.it/privacy/pubblicazione-delle-sentenze-e-privacy.html
} 
procedure tra stati diversi, cercando di eliminare in tal modo le differenze tra le legislazioni delli stati, che sono la prima causa di incertezza.

L’ interconnessione fra diritto della privacy e gli altri diritti costituzionali: il diritto all'informazione, il diritto di difesa e il diritto della pubblicazione delle sentenze, a nostro parere, trova una regolamentazione giuridica vera e propria nella legislazione italiana.

Questi sono le conclusioni che abbiamo tratto dallo studio ed in fine, visto la gamma molto ampia dellargomento, esce come raccomandazione l'eseguire di un altro studio, che studiera le altre problematiche associate con il diritto della privacy.

\section{References}

Mantelero, A., (2008), Contratto e impresa, Dialoghi con la giurisprudenza civile e commercial, CEDAM

Omari, L. \& Anastasi, A., (2010), E Dejta Kushtetuese, ABC

Cardaci, F. \& Olivetti, S., (1997), Diritto Civile, Il diritto alla riservatezza in Italia

Convenzione per la salvaguardia dei Diritti dell'Uomo e delle Libertà fondamentali

Carta dei Diritti Fondamentali dell'Unione Europea

Costituzione della Repubblica Italiana

Costituzione della Repubblica Albanese

http://brunosaetta.it/privacy/privacy-o-diritto-alla-riservatezza-e-protezione-dei-dati-personali.html

https://it.wikipedia.org/wiki/Privacy\#Fonti_comunitarie

http://brunosaetta.it/privacy/privacy-la-riforma-della-ue-e-il-do-not-track-standard.html

http://brunosaetta.it/privacy/pubblicazione-delle-sentenze-e-privacy.html 